\title{
THE EUROPEAN LOCATION FRAMEWORK - FROM NATIONAL TO EUROPEAN
}

\author{
E. Pauknerova ${ }^{\text {a }}$, P. Sidlichovsky ${ }^{\text {b }}$, S. Urbanas ${ }^{\text {c }}$, M. Med ${ }^{\text {a }}$ \\ ${ }^{a}$ Czech Office for Surveying, Mapping and Cadastre, Pod sidlistem 9, Prague, Czech Rep. - (eva.pauknerova, michal.med)@ cuzk.cz \\ ${ }^{\mathrm{b}}$ Land Survey Office, Pod sidlistem 1800/9, 18211 Prague 8, Czech Republic - pavel.sidlichovsky@cuzk.cz \\ ${ }^{\mathrm{c}}$ EuroGeographics, Rue de Nord 76, 1000 Brussels, Belgium - saulius.urbanas@eurogeographics.org
}

\section{Commission IV, WG IV/4}

KEY WORDS: GSDI, ELF, Harmonisation of Spatial Data, Interoperable Services, INSPIRE in Practice

\begin{abstract}
The European Location Framework (ELF) means a technical infrastructure which will deliver authoritative, interoperable geospatial reference data from all over Europe for analysing and understanding information connected to places and features. The ELF has been developed and set up through the ELF Project, which has been realized by a consortium of partners (public, private and academic organisations) since March 2013. Their number increased from thirty to forty in the year 2016, together with a project extension from 36 to 44 months. The project is co-funded by the European Commission's Competitiveness and Innovation Framework Programme (CIP) and will end in October 2016. In broad terms, the ELF Project will deliver a unique gateway to the authoritative reference geospatial information for Europe (harmonised pan-European maps, geographic and land information) sourced from the National Mapping and Cadastral Authorities (NMCAs) around Europe and including transparent licensing. This will be provided as an online ELF web service that will deliver an up-to-date topographic base map and also as view \& download services for access to the ELF datasets. To develop and build up the ELF, NMCAs are accompanied and collaborate with several research \& academia institutes, a standardisation body, system integrators, software developers and application providers. The harmonisation is in progress developing and triggering a number of geo-tools like edge-matching, generalisation, transformation and others. ELF will provide also some centralised tools like Geo Locator for searching location based on geographical names, addresses and administrative units, and GeoProduct Finder for discovering the available web-services and licensing them. ELF combines national reference geo-information through the ELF platform. ELF web services will be offered to users and application developers through open source (OSKARI) and proprietary (ArcGIS Online) cloud platforms. Recently, 29 NMCAs plus the EuroGeographics their pan-European umbrella association, contribute to the ELF through an enrichment of data coverage. As a result, over 20 European countries will be covered with the ELF topo Base Map in 2016. Most countries will contribute also with other harmonized thematic data for viewing or down-loading. To overcome the heterogeneity of data resources and diversity of languages in tens of European countries, ELF builds on the existing INSPIRE rules and its own coordination and interoperability measures. ELF realisation empowers the implementation of INSPIRE in Europe and it complements related activities of European NMCAs, e.g. Czech Office for Surveying, Mapping and Cadastre (CUZK), which provides a large portfolio of spatial data/services and contributes significantly to the NSDI of Czech Republic. CUZK is also responsible for the Base Register of Territorial Identification, Addresses and Real Estates (RUIAN) - an important pillar of Czech e-Government. CUZK became an early-bird in implementing INSPIRE and it provides to the ELF a number of compliant datasets and web services. CUZK and the Polish NMCA (GUGiK) collaborate in the Central-European ELF Pilot (cluster) and test various cross-border prototypes. The presentation combines the national and crossborder view and experiences of CUZK and the European perspective of EuroGeographics.
\end{abstract}

\section{INTRODUCTION}

\subsection{Diversity and Interoperability Trends in Europe}

Europe is a relatively heterogeneous continent. There are 50 internationally recognized sovereign states with territory located within the common definition of Europe and/or membership in international European organisations, incl. EuroGeographics (EG). 28 of these countries have also been Members of the European Union since and sharing a part of their suvereignity with EU institutions. There are 24 official languages at the EU. Any diversity can provide inspirations, healthy competition, and ecosystem's resistence. On the other hand, there are challenging barriers or potential conflicts.Therefore, establishing and empowering interoperability became a significant aim, esp. in the digital era. There are various formal programmes (ISA), legal documents (INSPIRE Directive and its Implementing
Rules), actions (ELISE), international initiatives or technical standards (CEN, OGS) and other tools used to increase interoperability among the EU member states, their public administration and their services to support mobility and single market at the EU+. International projects as ELF provide a good environment for a proof of concept, exchange of experiences, pilot development and for setting up prototypes.

The ELF project is a large European pilot ${ }^{1}$, enforcing interoperability of geospatial data and related webservices. It builds on the content provided by NMCAs. Forteen countries were represented initially and 29 NMCAs - from 25 countries -

\footnotetext{
${ }^{1}$ The Competitiveness \& Innovation framework Programme (CIP) Information and Communication Technologies Policy Support Programme (ICT PSP) Open Data and open access to scientific information (Project reference 325140)
} 
are involved or collaborating since 2016. Strategic planning and preparations by EuroGeographics and its members started in 2015 to ensure ELF's sustainability and further development beyond the ELF project.

\subsection{Definitions}

Several terms were specified for purposes of ELF, as below. For more info, see http://elfproject.eu/documentation/glossary.

Authoritative data: Data that are kept and updated by a trusted source and used through the national spatial data infrastructure in the variations on scale and details required.

Reference data: Geospatial data, which gives an unambiguous location for a user's information by providing a geographic context which enables merging of data from various sources.

ELF product specification: Specification based on the ELF data specifications providing a detailed description of a dataset or dataset series together with additional information that will enable the datasets to supplied to and used by another party through an ELF service.

\subsection{ELF and Other EU Initiatives}

The ELF builds of knowledge and outputs which had been developed during the previous ESDIN project and it provides a 'proof of concept'. It also further develops the tools and data content of some EuroGeographics' pan-European Products, e.g. ERM, EGM.

The INSPIRE Directive, with its Implementation Rules and related Technical Guidelines, see http://inspire.ec.europa.eu/; provide an important formal framework established at the EU and respected also by other countries in Europe as regards SDI. This entire legislation and related Road Map outline the direction, timeframe and structure of arrangements to be done in all EU MSs till 2021. Nevertheless, INSPIRE implmenetation is extremely complex and if not systematically coordinated and cross-checked, it might fail easily. To reach seamless data and services across Europe and their sharing among organisations and between local, national and European levels or with other stakeholders and users, it requires additional efforts, tools and collaboration among MSs.

ELF is based on INSPIRE and it provides a subset of INSPIRE specifications with some additional features needed for crossborder and pan-European interoperability. The ELF project has been proposing these to be included in the future development and maintenance of INSPIRE specifications.

ELF Project aims to be complementary or to establish mutual cooperation with other European initiatives as Copernicus, UN-GGIM: Europe or EULF /ELISE as regards ISA and egovernment, as already noticed in the section on Geospatial Solutions at the ISA2 Work Programme 2016. In some ways ELF can be seen as a strong showcase for the e-government in Europe.

\subsection{What makes ELF unique?}

ELF is based on national level geospatial data services and creates a single harmonised European service that will work seamlessly across country borders. It addresses the dual need for a simple, easy-to-use web maps to integrate into a website or an application and providing access to datasets to download for those wishing to work with the data.

ELF complements to NMCAs' activities dealing with the INSPIRE implementation at the national level. With its focus on cross-border harmonisation of reference data, their quality assurance and on international and pan-European products it differs from and complements also to the function of the INSPIRE Euro Geoportal (maintained by the EC JRC).

\subsection{ELF Goal and Objectives}

The goal of this project is to deliver the European Location Framework (ELF) required to provide up-to-date, authoritative, interoperable, cross-border, reference geo-information for use by the European public and private sectors. This versatile cloud-based and cascade-supporting architecture provides a platform of INSPIRE compliant geo-information, harmonised at a cross-border and pan-European level.

The objectives of the project are to:

add value to INSPIRE data by contributing to cross border harmonisation

build a high performance platform and associated cloud services that support multiple national feeds and a wide spectrum of value-added services

- demonstrate the usability of the ELF platform and cloud services for key European policy areas and other users

- develop sample applications in the sectors of Health Statistics, Emergency Mapping, Insurance

- collaborate with 3rd parties and NSDIs to integrate thematic datasets beyond the INSPIRE themes provided by NMCAs for service implementations based on specific user needs

- provide a user friendly interface to find, view and compare the geo-information

\section{METHODS AND RESULTS}

\subsection{Methodology}

The ELF Project is a large and complex project requiring the coordinated work of 40 consortium partners. To reach its longterm goal and project objectives, the ELF Consortium set up its organisational structure, formal arrangements and communication tools. The key roles of Project Coordinator and Project Administrator are supplied by Kartverket (Norway) and of Technical Coordinator by the National Land Survey, Finland. In order to manage the delivery of the project this work is divided into 9 sub-projects, referred to as Work Packages (WP):

\footnotetext{
WP 1: Management of the ELF Project (led by Kartverket)

WP 2 : ELF Specifications (led by BKG, Germany)

WP 3 : ELF Services and Data Content (led by Kartverket)

WP 4 : Implementation of ELF Geo-Tools (led by Dutch Kadaster)

WP 5 : ELF Cloud Service Platform (led by interactive instruments)

WP 6 : User and 3rd party data content for ELF (led by EuroGeographics)
} 
WP 7 : ELF Service Instances (led by the National Land Survey, Sweden)

WP 8: ELF Engagement and Dissemination (led by Netrius Management Services, UK)

WP 9: Sustainable access and use of ELF services (led by Kartverket)

See http://www.elfproject.eu/content/structure

ELF applied the ESDIN concept and specifications and builds on the INSPIRE legal fundament (which considers and therefore puts more weight to international technical standards as concerns GI and SDI).

Numerous surveys were done by WP3 and WP9 among the project partner organisations in order to map the state of play (e.g. mapping availability and conformity of data and services, existing business models and licencing policy for their provision). The user needs were in focus of questionnaires and consultation done by WP6 and WP7 with $3^{\text {rd }}$ parties and in specific user domain, esp. by WP3, WP6, WP7 and WP9.

There were also several types of (sub)pilot activities to implement and test prototypes in their initial phase and to provide feed-back for new versions of Data or Product Specifications or their broader implementation:.

Two clusters (Scandinavian countries, esp. FI, NO and SE, and Central European - PL, CZ and partially DE) / data harmonisation, early stage implementation of the new ELF base maps (topographic and cadastral index map - C.I.M).

- Thematic use cases and sub-pilots of WP6 (DEM30, IO) and Thematic applications development and testing by WP7.

Different sorts of independent testing have been realized and still foreseen during the project, e.g. with focus on service performance, data or service compliance, correspondence to user-needs.

Several types of training and awareness raising activities and events (workshops, webinars) have been organized by most WPs for both audiences, project stakeholders or specialists and external partner organisations or potential users.

For a mutual understanding (semantic interoperability) within such a large multilingual team a joint terminology had to be defined and documented in the ELF Glossary.

In order to build up the entire ELF infrastructure, several types of elements had to be focused, specified, developed/designed, implemented, tested, modified (in some cases). Their future sustainable performance and availability needs to be provided.

The centre of the technical architecture of ELF is created by the ELF Platform (http://locationframework.eu). An intensive attention was paid by WP2 (in collaboration with WP3 and WP4) to the development of several sorts of ELF Specifications. These are Specification for:

Data Maintenance and Processing

ELF Cadastre (or Cadastral Index MAP - CIM)

ELF Base Map

ELF Data Specifications

ELF Data Model

ELF International Boundaries

\section{Service specifications for ELF Geo Locator}

Various types of tools were needed to support harmonisation efforts of NMCAs to reach interoperability of their products and new ELF services at several levels. Geo-tools were or are being developed by academia institutes or specialized private companies to empower NMCAs' activities as edge matching, generalisation, change detection, quality assurance, transformation, visualisation etc.

Interconnections among different components of the ELF infrastructure are displayed at the scheme below (Figure1).

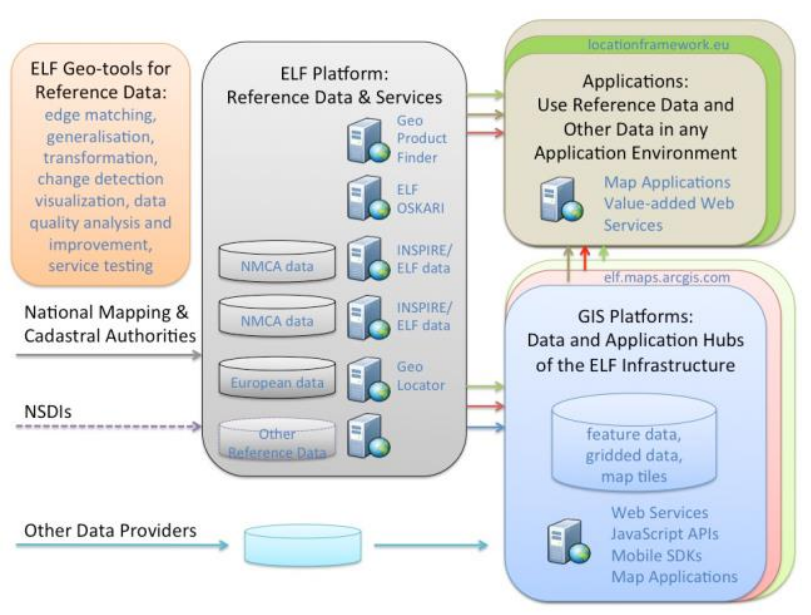

Figure 1. The ELF Platform and Elements of ELF Architecture

ELF combines national reference geo-information through the cloud based ELF infrastructure and by combination of national download and view services it will provide a new Base Map service and thematic data coverages for Europe. ELF will move data to services in a European SDI as envisioned by the INSPIRE Directive,

The participating NMCAs harmonise their national data and services in accord with the common pan-European specifications (INSPIRE, mostly Annex I and II Themes) and/or in compliance with the ELF Specifications. In this way they contribute to the creation of new ELF WMS services and a seamless pan-European coverage of topographical or thematic data.

Data providers planning to add their data to the ELF Platform need (in order to maintain the authoritative nature of ELF), to adhere to the ELF data specifications. They can use the ELF GeoTools to ensure that data quality standards are met. Such data transformation must happen before the data is then fed into the ELF infrastructure. Data providers have no obligation to make their data available through ELF but a key objective of the project is to ensure that their commercial and licensing rights are preserved if they do.

The Czech Office for Surveying, Mapping and Cadastre with its specialized body - the Land Survey Office (ZU), have been maintaining and providing a large portfolio of spatial data and services. These concern both, topography and location or territory related administration. The tools and methods of 
photogrammetry and remote sensing (P\&RS) represent an integral part of data acquisition and up-dating. P\&RS based products are published and made accessible via the CUZK Geoportal: http://geoportal.cuzk.cz/geoprohlizec/?wmcid=13993

CUZK products and services create a significant part of the National Spatial Data Infrastructure (NSDI) in the Czech Republic (CZ). They also serve as a stable source of CZ data sets and related services for the Infrastructure for Spatial Information in the European Union (INSPIRE).

CUZK is responsible for the Base Register of Territorial Identification, Addresses and Real Estates (RUIAN), which represents one of four Base Registers of Czech e-Government. Since July 2012, RUIAN has been providing up-to-date location data for $\mathrm{CZ}$ public authorities. In addition, a public access via the Internet enables a broad re-use of these elementary location data in numerous user oriented applications.

The timing of the RUIAN development put CUZK into an early-bird position as regards setting-up the INSPIRE Implementing Rules into practice. CUZK together with ZU provide INSPIRE conform data sets and web services for most of the INSPIRE Themes contained in Annexes I and II, see http://geoportal.cuzk.cz/Default.aspx? Ing $=E N \&$ mode $=$ TextMet $a \&$ side $=w m s . I N S P I R E \&$ text $=$ WMS.INSPIRE\&head_tab $=$ sekce -03-gp\&mеnи $=313$.

CUZK has been contributing to the ELF project with its INSPIRE-implementation experiences and a number of harmonized products.

Together with the Polish GUGiK, CUZK contributes through various pilot activities at the Central-European Cluster, as:

data availability analyses and cross-border interoperability testing done in WP3 in 2014;

WP6 sub-pilot lead by BKG on development of the EuroDEM30 prototype to be tested against requirement of Euro Control.

CUZK also was involved in the preparations and early implementation and prototype testing of both new ELF base maps - the topo Base Map and the Cadastral Index Map.

\subsection{ELF Results}

The ELF project has set up a technical framework on which future location services can be built. It has overcome a number of technical challenges, and built a technical infrastructure that can deliver pan-European location services. This includes harmonisation standards and tools. Project information, including documentation becomes available via ELF project website, see http://elfproject.eu/documentation.

The number of NMCAs which have been involved in the project and signed up to provide data content and interoperable services was enlarged - from 14 consortium members in 2013 to 24 in 2016, and other 4 data providers collaborate outside the project. See Fig 2,

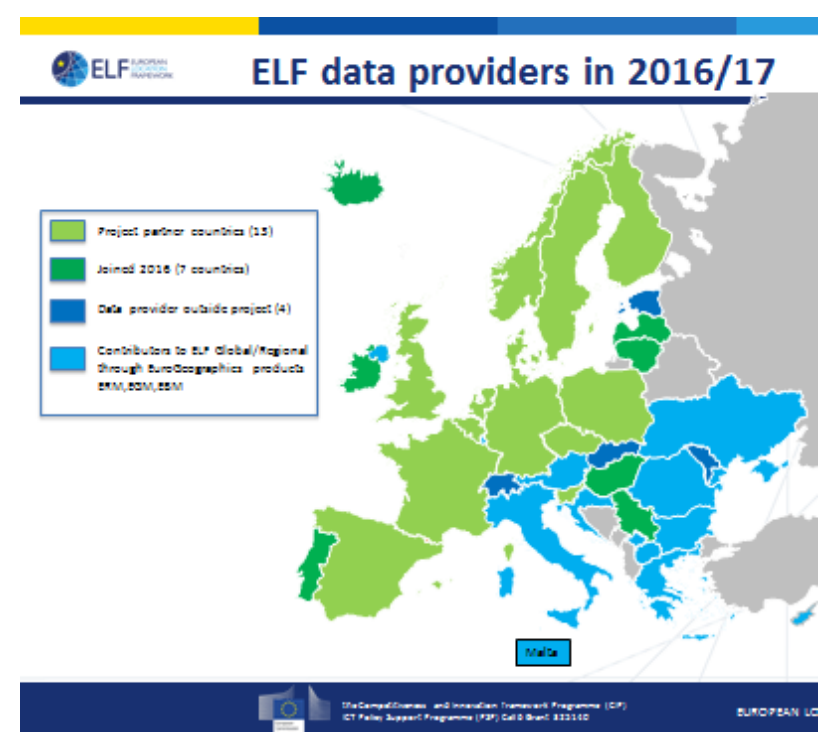

Figure 2. ELF Data Providers (as agreed in 2016)

When this paper is being delivered, the ELF infrastructure has not been available for a standard, public use yet. Nevertheless at this phase, the ELF Project has been finalizing and completing most of its Deliverables defined in the Description of Work document (DoW). These are: ELF Platform, ELF Base Map Service, ELF Cadastral Index Map, ELF Geo Product Finder, ELF View and Download Services, ELF Geo Locator etc.

Following Figures illustrate results of prototype activities realized in Central Europe in the years 2015 and 2016.

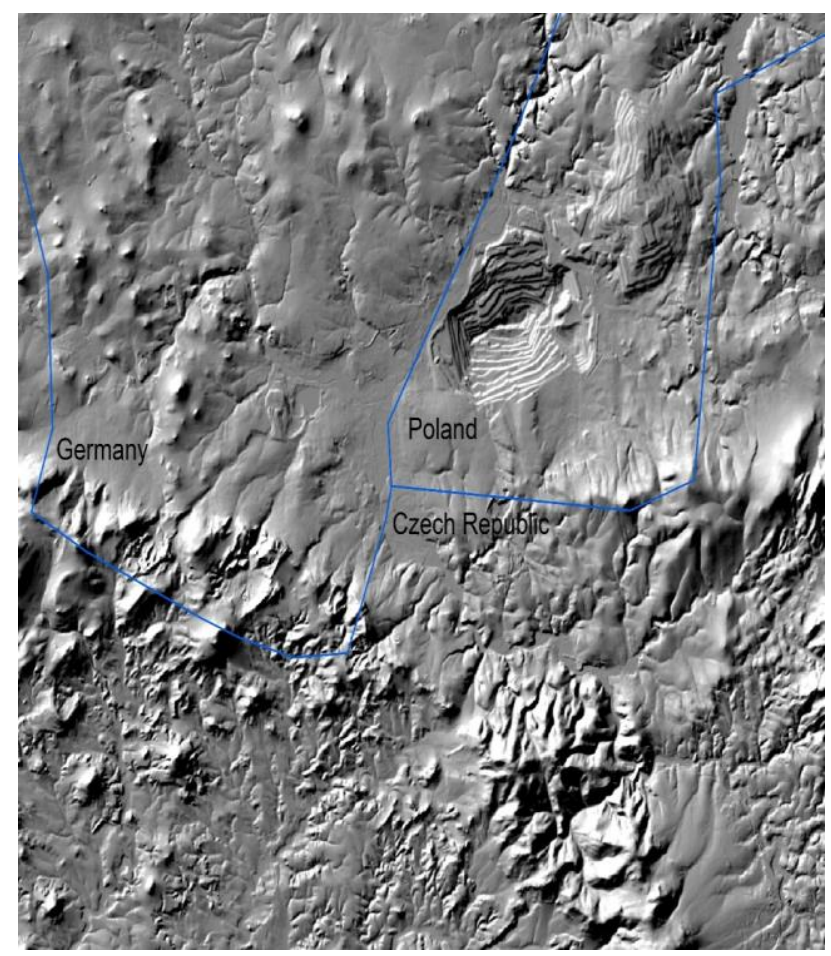

Figure 3. EuroDEM30 Pilot (DE-PL-CZ case study based on data provided by $B K G, G U G i K$ and $C U Z K-Z U)$ 
Pilot dataset for production of the Digital Elevation Model DEM30 based on the requirements of Euro Control (TOD Terrain and Obstacle Data) contained data from Germany, Poland and a buffer zone following borders of Czech Republic. The Digital Terrain Model with a grid width of 1 arc second was produced in cooperation of German BKG, Polish GUGiK and Czech ZU and harmonized at country borders, (Figure 3). Heights were derived by Laser-Scanning and StereoPhotogrammetry. Grid width 1 arc second corresponds to $30 \mathrm{~m}$ in meridian direction. 1-3 meters vertical accuracy was achieved. Geodetic Datum: ETRS89, Vertical Datum EVRS. Evaluation done from the perspective of aeronautical applications brought positive results.

Czech with the Polish NMCAs have also collaborated on crossborder prototypes connected with the development of ELF base maps /- the topo Base Map and the Cadastral Index Map. These activities concerned both, data harmonisation (visualisation, generalisation, edge-matching etc.) and assurance of interoperable webmap services provided by CUZK and GUGiK. Figure 4 illustrated a combination of prototypes of the ELF Base Map with the C.I.M. in an area near the Czech-Polish border.

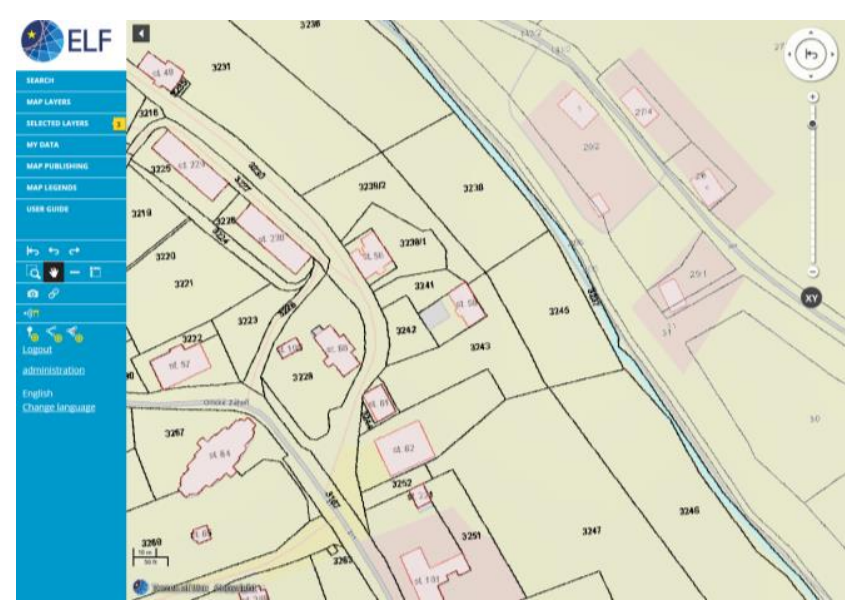

Figure 4. Cadastral Index Map (C.I.M.) underpinned by the ELF topo Base Map - Czech-Polish border

The Figure 4, shows (in a simplified way) results of two rather complex activities realized by WP2 and WP3 and implemented and tested in both the cluster regions of ELF.

The new ELF Base Map (developed under lead of IGN France) is a pyramid of digital maps, at different zoom levels, built from the ELF harmonized data of NMCAs. At large scales primary source are the INSPIRE themes AU, GN, HY, TN, EL, BU, LC and BU. Smaller scales pyramid levels are derived from data of European Regional Map (ERM) or Euro Global Map (EGM) which are products of EuroGeographics. The map represents only entities currently in existence. National Base Map services are published as WMS for the purpose of map caching by servers at the Norwegian Kartverket, which then publishes single central Base Map service as WMTS, structured into 24 zoom levels. The purpose of the service is mainly to provide reliable topographic map, to be used as A background map for other applications such as websites, GIS and mobile devices.
The ELF Cadastre, described also as the ELF Cadastral Index Map (C.I.M.) is a new view service developed by ELF Project under lead of SDGC (Spain). C.I.M. provides a simplified and harmonised view of footprints of cadastral parcels geometry combined with other basic information as administrative units, addresses and buildings for pan-European use. It will permit also to identify the features and to obtain both the national cadastral reference of the cadastral parcel, that gives users the opportunity to get more information through the cadastral national services, and the complete address of the cadastral parcel or building. This specification provides: A narrative description of the ELF Cadastral Index Map service, an example of the data content and basic guidelines for implementation. The main chapter is the portrayal one that defines the ELF Cadastral Index Map style taking into account the INSPIRE default portrayal for themes CP, BU, AD and AU, the combination of all of them and the range of scales of ELF Cadastral Index Map.

The C.I.M. sample at Figure 4 looks simple or boring from a cartographic point of view, but it represents an achievement from the data maintenance and quality perspective. The C.I.M. web service has been provided by CUZK to the ELF with regular daily up-dates. Related themes - CP, AD, AU and BU are edited continuously by about 8000 local authorities (via RUIAN and eGovernment as a part of municipal, building or statistical agendas). Based on such fresh content provided by RUIAN, CUZK generates datasets harmonized for relevant themes in an extent of the whole country. These national datasets are provided as interoperable WMS or WFS to the INSPIRE. In case of the C.I.M. web service, the fresh authoritative content - edited by local experts and officers becomes an integral part of the seamless ELF webmap on the very same day. In this way, it contributes to its reliability.

There were also some secondary results. Finding of prototype implementations provided a useful feed-back not only to the ELF WP2 working on the ELF Specifications. Information about specific inconsistences or implementation issues regarding INSPIRE which had been detected during the ELF project was addressed also to the INSPIRE coordination bodies, e.g. EC JRC, INSPIRE Thematic clusters and the MIG-T. Proposals for consequent modifications or arrangements were debated at different levels of this coordination framework consequently.

The ELF Project is now focussing on providing some test services before it ends in October 2016. The ELF platform will not be available to users outside the project consortium until autumn 2016. There is an ELF Showcase application today (at www.locationframework.eu) which allows a user to see ELF in use. In November 2016 the project will enter a two-year 'transition' phase under the 'ownership' of EuroGeographics,

\section{CONCLUSIONS}

Although the ELF did not reach its full operational functionality, most of its deliverables have been functional at least in a prototype mode. These are exposed or will be provided to different sorts of testing soon.

Already in this phase, ELF can demonstrate several potential benefits via its pilot cases, e.g.: 
- A single source of official, quality-assured data from Europe's national mapping, cadastral and land registry authorities.

- Harmonised data delivered to a common and consistent specification.

The large number of participating organisations provided a challenging environment and a variety of issues to be solved by the management bodies and/or participants themselves. On the other hand such an international, multidisciplinary framework enables sharing of experiences, lessons learned; it offers new inspiration for members and the new comers. The positive experience of participating NMCAs can be used to encourage other NMCAs from across Europe to become involved.

Findings made through the implementation may contribute to enhancements of methods to be applied further (in INSPIRE or ELF). Unexpected barriers invoke new tasks (e.g. ETRS boundaries as stated by Polacek, J.) and their future solution within the project or with new partners outside the project.

After several years, the original concepts need to be reevaluated regarding external impacts and other risks considered, if needed. The established infrastructure provides a good basis enabling to do so. Important is a shared belief that despite all the difficulties met on the road the European Location Framework enabling future functional services is a good idea and a need to make it happen.

\section{REFERENCES}

Information provided in this paper is based mostly on:

a) materials of the ELF project; at this stage partially published via http://elfproject.eu; e.g.

http://elfproject.eu/documentation/glossary

b) documents of the European Commission on INSPIRE or

ISA, e.g.

http://inspire.ec.europa.eu/index.cfm/pageid/3

c) Information published via CUZK geoportal, e.g. http://geoportal.cuzk.cz/; e.g.

http://geoportal.cuzk.cz/geoprohlizec/?wmcid=11511.

http://geoportal.cuzk.cz/Default.aspx?lng $=E N$,

http://geoportal.cuzk.cz/Default.aspx?Ing $=E N \&$ mode $=$ TextMeta\& side $=$ wms.INSPIRE\&text $=$ WMS.INSPIRE\&head_tab $=$ sekce-03gp\&menu $=313$.

d) conference abstracts or presentations, e.g.

Polacek, J., 2015, State Boundaries in ETRS89

Coordinates - Reality or Fiction,

http://geospatialworldforum.org/speaker/bio-

abstratct.asp?id=gwf2015 A365 